\title{
Layer-by-layer assembly of polycations and polyanions for the sensitive detection of endotoxin
}

\author{
Biyas Posha, Haritha Kuttoth, N Sandhyarani*
}

Nanoscience Research laboratory, School of Material Science and Engineering, National Institute of Technology Calicut 673601, Kerala, India, Fax: 91495 2287250; Tel: 91495 2286537

E-mail: sandhya@nitc.ac.in

Au electrode cleaning in $\mathrm{HClO}_{4}$ by potential cycling from $0.2 \mathrm{~V}$ to $1.5 \mathrm{~V}$.

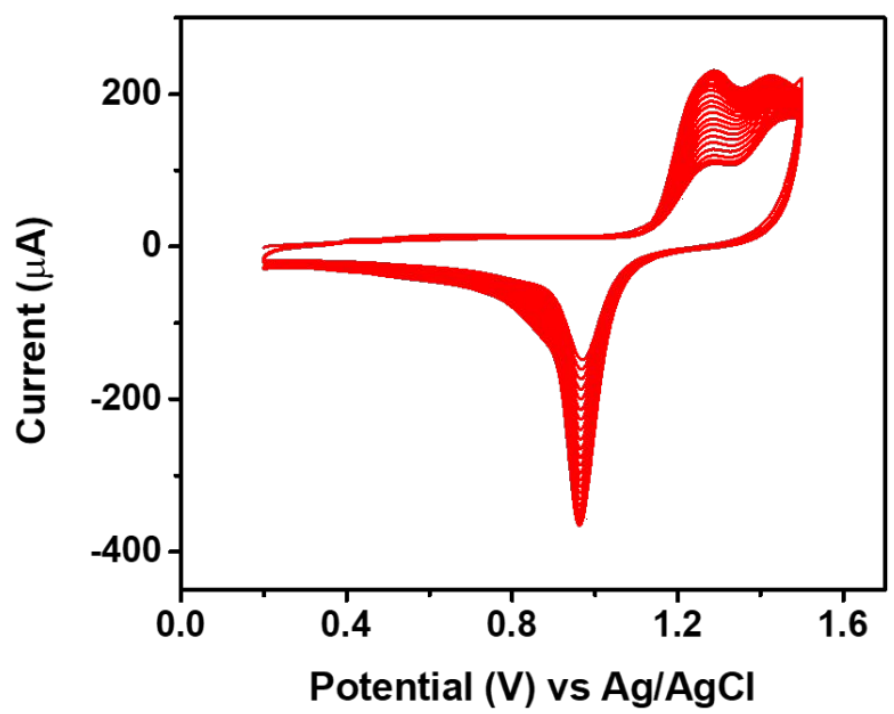

Figure S1 Potential cycling from $0.2 \mathrm{~V}$ to $1.5 \mathrm{~V}$ at a scan rate of $100 \mathrm{mV} \mathrm{s}^{-1}$ of the $\mathrm{Au}$ electrode in $0.1 \mathrm{M} \mathrm{HClO}_{4}$ for electrochemical cleaning. 
UV- Visible spectra of CGNC, PAA/CGNC and PmB/PAA/CGNC.

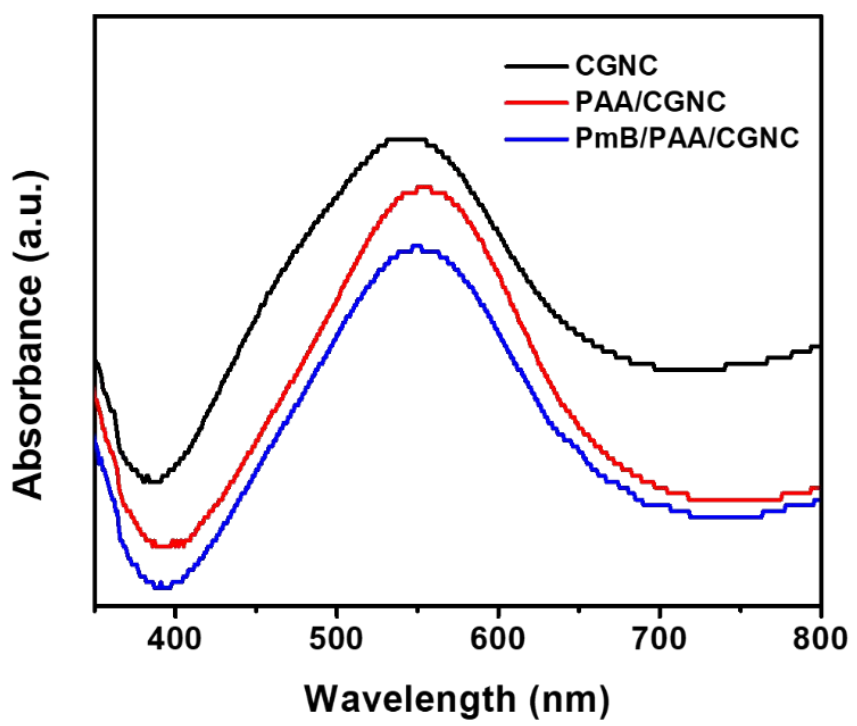

Figure S2 UV-Visible spectra of CGNC, PAA/CGNC, and PmB/PAA/CGNC.

FT-IR spectra of CGNC/Au, PAA/CGNC/Au, and PmB/PAA/CGNC/Au.

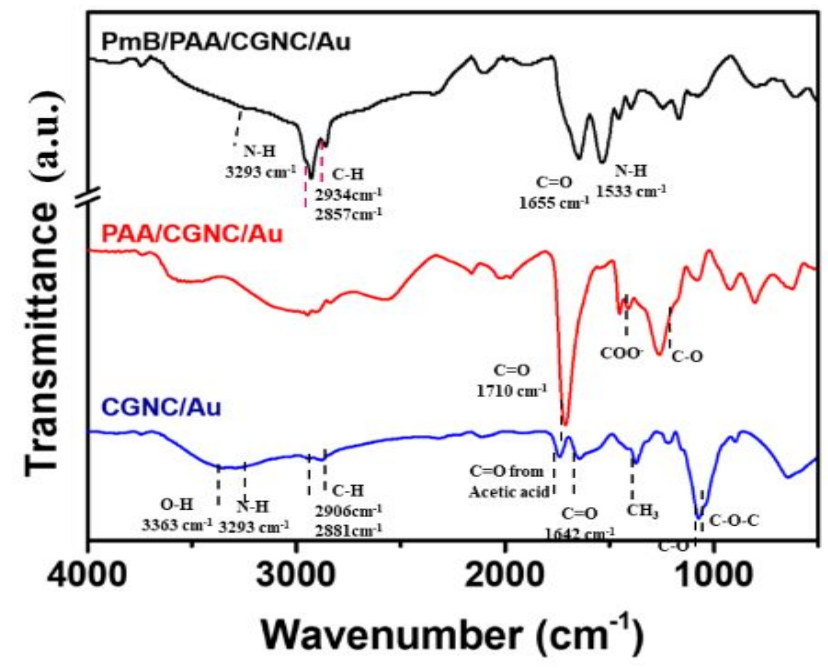

Figure S3 FT-IR spectra of CGNC/Au, PAA/CGNC/Au and PmB/PAA/CGNC/Au. 
EIS equivalent circuit elements values

Table S1 Equivalent circuit element values for the different modification of the electrode: $\mathrm{Au}$ electrode, $\mathrm{CGNC} / \mathrm{Au}, \mathrm{PAA} / \mathrm{CGNC} / \mathrm{Au}$, and $\mathrm{PmB} / \mathrm{PAA} / \mathrm{CGNC} / \mathrm{Au} . \quad$ Electrolyte - 5 $\mathrm{mM} \mathrm{K}_{3}\left[\mathrm{Fe}(\mathrm{CN})_{6}\right]$ in $0.1 \mathrm{M} \mathrm{KCl}$.

\begin{tabular}{|c|c|c|c|c|c|c|}
\hline $\begin{array}{c}\text { Electrode } \\
\text { modification }\end{array}$ & $\begin{array}{c}\mathrm{R}_{\mathrm{s}} \\
(\Omega)\end{array}$ & $\begin{array}{c}\mathrm{C}_{\mathrm{dl}} \\
(\mathrm{F})\end{array}$ & $\begin{array}{c}\mathrm{R}_{\mathrm{ct}} \\
(\Omega)\end{array}$ & $\begin{array}{c}\mathrm{Q} \\
(\mathrm{F})\end{array}$ & $\begin{array}{c}\mathrm{R}_{\mathrm{dl}} \\
(\Omega)\end{array}$ & $\begin{array}{c}\mathrm{W} \\
(\Omega)\end{array}$ \\
\hline $\mathrm{Au}$ electrode & 5.47 & $6.37 \mathrm{E}-9$ & 9.18 & $\begin{array}{c}1.17 \mathrm{E}-3 \\
(\mathrm{n}=0.58)\end{array}$ & 6.96 & $6.9 \mathrm{E}-17$ \\
\hline $\mathrm{CGNC} / \mathrm{Au}$ & 4.68 & $5.67 \mathrm{E}-8$ & 12.34 & $\begin{array}{c}4.37 \mathrm{E}-3 \\
(\mathrm{n}=0.44)\end{array}$ & $4.2 \mathrm{E} 4$ & $4.7 \mathrm{E}-16$ \\
\hline $\begin{array}{c}\mathrm{PAA} / \mathrm{CGNC} / \\
\mathrm{Au}\end{array}$ & 6.45 & $3.33 \mathrm{E}-8$ & 20.46 & $\begin{array}{c}3.57 \mathrm{E}-4 \\
(\mathrm{n}=0.70)\end{array}$ & 289.9 & 618 \\
\hline $\begin{array}{c}\mathrm{PmB} / \mathrm{PAA} / \mathrm{C} \\
\mathrm{GNC} / \mathrm{Au}\end{array}$ & 4.82 & $3.32 \mathrm{E}-8$ & 25.66 & $\begin{array}{c}3.0 \mathrm{E}-4 \\
(\mathrm{n}=0.78)\end{array}$ & 95.76 & $1.9 \mathrm{E}-3$ \\
\hline
\end{tabular}

Cyclic voltammogram of $\mathrm{Au}$ electrode, $\mathrm{CGNC} / \mathrm{Au}, \mathrm{PAA} / \mathrm{CGNC} / \mathrm{Au}$ and $\mathrm{PmB} / \mathrm{PAA} / \mathrm{CGNC} / \mathrm{Au}$ in PBS.

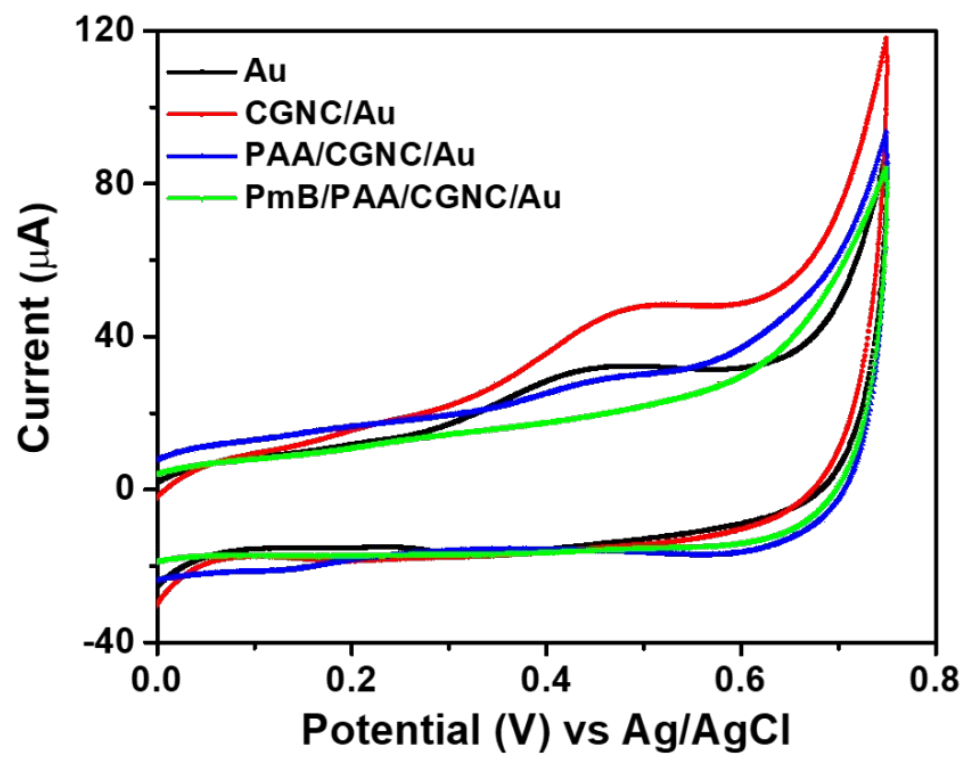

Figure S4 Cyclic voltammogram of Au electrode, CGNC/Au, PAA/CGNC/Au and $\mathrm{PmB} / \mathrm{PAA} / \mathrm{CGNC} / \mathrm{Au}$ in PBS. 
DPV plots of different concentrations of LPS at CGNC/Au

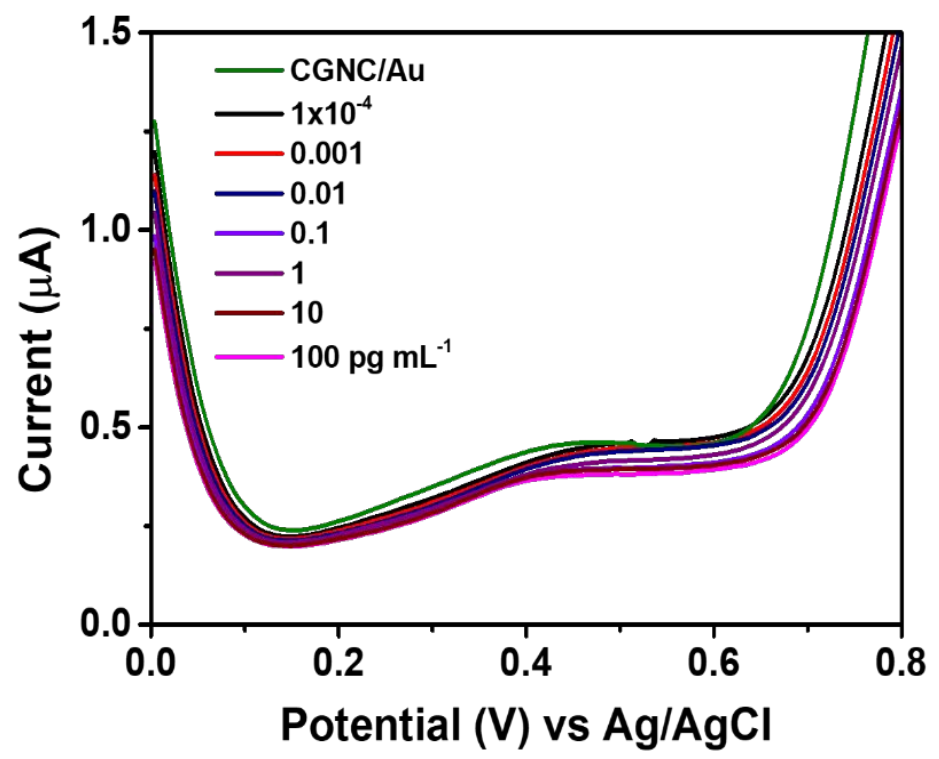

Figure S5 DPV plots of various concentration of LPS (100 ag mL $\mathrm{mL}^{-1}$ to $\left.0.1 \mathrm{ng} \mathrm{mL}^{-1}\right)$ at CGNC/Au in $0.1 \mathrm{M}$ PBS.

DPV analysis of whole blood sample by standard addition method and corresponding calibration plot
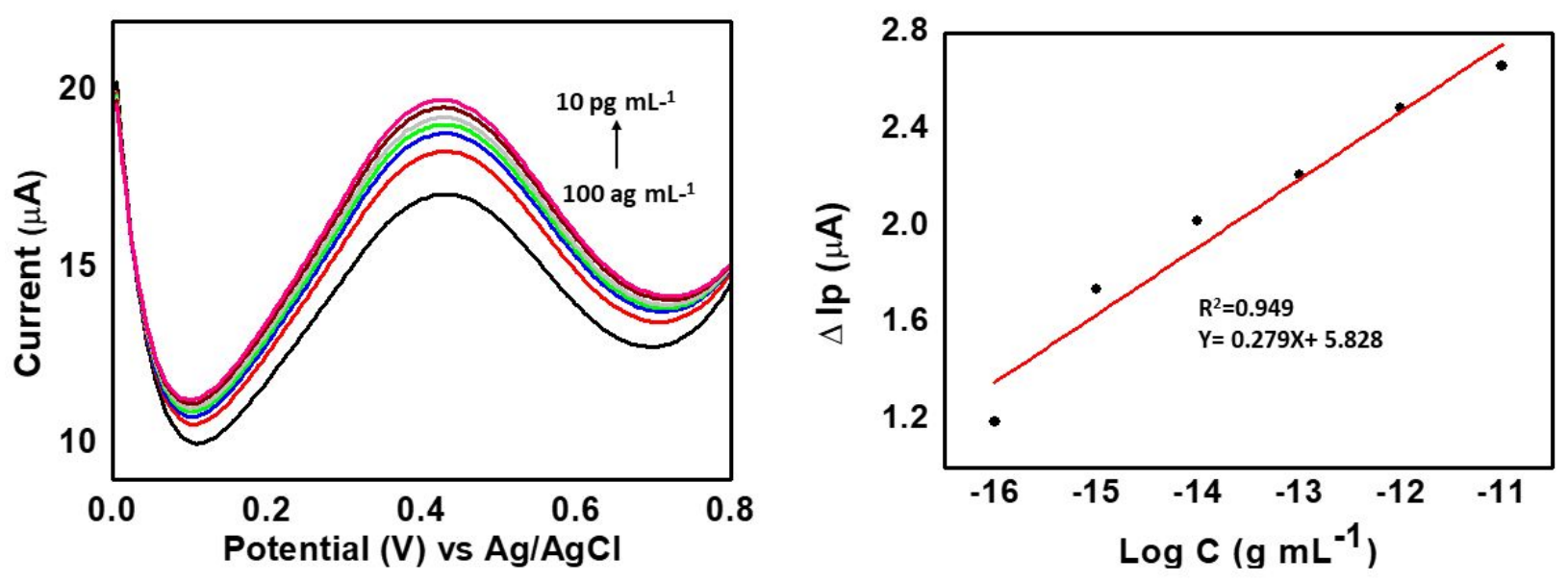

Figure S6 DPV plots of PmB/PAA/CGNC/Au incubated in whole blood in 0.1 M PBS and different concentrations of LPS (100 ag $\mathrm{mL}^{-1}$ to $\left.100 \mathrm{pg} \mathrm{mL}^{-1}\right)$ in $0.1 \mathrm{M}$ PBS containing whole blood, (B) corresponding calibration plot of $\Delta \mathrm{Ip}$ vs logarithm value of LPS concentration. 